\title{
EFEITO DA ESPESSURA DA POLPA, TAMANHO E PESO DE FRUTOS DE TAPEREBÁ (Spondias mombin L.) SOBRE O PARASITISMO NATURAL. (HYMENOPTERA: BRACONIDAE) EM MOSCAS-DAS-FRUTAS (DIPTERA: TEPHRITIDAE)
}

\author{
Alan Cavalcanti da Cunha ${ }^{1}$, Ricardo Adaime da Silva ${ }^{2}$, Júlia Daniela Braga Pereira ${ }^{3}$, Rodrigo \\ Souza Santos ${ }^{4}$
}

'Universidade Federal do Amapá, Curso de Ciências Ambientais, Programa de Pós-graduação em Biodiversidade Tropical, Rodovia JK, Km 4, 68902-280, Macapá, Amapá, Brasil.

2E mbrapa Amapá, Rodovia JK, km 5, n²600,68903-419, Macapá, Amapá, Brasil. adaime@cpafap.embrapa.br.

${ }^{3}$ Universidade Federal do Amapá/UNIFAP, Rodovia JK, Km 4, 68902-280, Macapá, Amapá, Brasil.

${ }^{4}$ Pós-doutorando PNPD/CAPES/FINEP. Embrapa Amapá, Rodovia JK, km 5, n²600, 68903-419, Macapá, Amapá, Brasil.

\section{RESUMO}

Os frutos do taperebazeiro (Spondias mombin L.) são muito apreciados pelas comunidades amazônicas, tanto para consumo in natura quanto em formas processadas. Do ponto de vista ecológico, é uma espécie que exerce importante função como reservatório de parasitóides (Hymenoptera) de moscas-das-frutas (Diptera: Tephritidae). De maneira geral, seus frutos são desuniformes em tamanho, peso e espessura de polpa. $\mathrm{O}$ objetivo deste trabalho foi verificar se esses parâmetros interferem na incidência de parasitóides de moscas-das-frutas. Foram coletados 75 frutos maduros (cada fruto individualmente representou uma amostra) de um taperebazeiro na área urbana do município de Macapá, Amapá. Os parâmetros analisados foram: peso dos frutos, maior e menor diâmetro dos frutos, espessura de polpa, índice de infestação de frutos por moscas-das-frutas e percentual de parasitismo. Não foi observada a existência de uma função matemática do tipo "decaimento exponencial" que relacionasse os parâmetros avaliados ao percentual de parasitismo. Houve, porém, uma tendência de decréscimo linear, com baixa significância estatística.

Palavras-chave: Tephritidae, Hymenoptera, Amapá, Amazônia

$$
\begin{aligned}
& \text { EFFECT OF PULP THICKNESS, SIZE AND WEIGHT, TAPEREBÁ FRUITS (Spondias } \\
& \text { mombin L.) ON THE NATURAL PARASITISM (HYMENOPTERA: BRACONIDAE) IN } \\
& \text { FRUIT FLY (DIPTERA: TEPHRITIDAE) }
\end{aligned}
$$

\begin{abstract}
Fruits of taperebá tree (Spondias mombin L.) are highly appreciated by Amazonian communities for both fresh and in processed forms consumption. It is a species with important function as reservoir of parasitoids (Hymenoptera) of fruit flies (Diptera: Tephritidae) from the ecological point of view. Overall, its fruits are irregular in size, weight and pulp thickness. The aim of this study was to determine whether these parameters influence the performance of fruit flies parasitoids. Exactly 75 ripe taperebá fruits (each fruit individually accounted for a sample) were collected in an urban area of Macapá, Amapá. Parameters analyzed included: fruits weight, larger and smaller diameter, pulp thickness, fruits infestation index and parasitism percentage. The existence of a mathematical function such as "exponential decay" relating evaluated
\end{abstract}


parameters to parasitism percentage was not observed. However, there was a trend of linear decrease with low statistical significance.

Key words: Tephritidae, Hymenoptera, Amapá, Amazon

\section{INTRODUÇÃO}

O taperebazeiro (Spondias mombin L.), também conhecido como cajazeiro, é uma frutífera caducifólia que apresenta 18 a $25 \mathrm{~m}$ de altura. Nativa da região Amazônica e da Mata Atlântica tem ampla ocorrência natural, sendo ocasionalmente cultivada em pomares domésticos. Seus frutos globosos ou elípticos, do tipo drupa, com polpa suculento-fibrosa, de sabor doce-acidulado, são consumidos frescos e na forma de sucos, sendo muito comercializados em feiras livres nas regiões Norte e Nordeste do Brasil (Lorenzi et al. 2006).

Entre os insetos associados ao taperebazeiro, são registradas espécies de moscas-das-frutas (Diptera: Tephritidae): Anastrepha antunesi Lima, Anastrepha distincta Greene, Anastrepha fraterculus (Wiedemann), Anastrepha obliqua Macquart, Anastrepha sororcula Zucchi, Anastrepha striata Schiner e Anastrepha turpiniae Stone (Zucchi 2008; Silva et al. 2011).

Estudos conduzidos no estado do Amapá têm demonstrado a importância do taperebá como planta hospedeira de espécies de moscas-das-frutas e, principalmente, como "reservatório" de parasitóides (Silva et al. 2007a). Lemos et al. (2008) coletaram 85 amostras de taperebá $(99,71 \mathrm{~kg})$ em quatro municípios do estado do Amapá e concluíram que as espécies de moscas-dafrtuas mais abundantes foram $A$. obliqua $(75,1 \%), A$. antunesi $(19,5 \%), A$. striata $(4,9 \%)$ e $A$. fraterculus $(0,5 \%)$. Também no Amapá, Silva et al. (2005) registraram os parasitóides Doryctobracon areolatus
(Szépligeti), 92,1\% do total de exemplares, e Opius bellus Gahan, 7,9\%, associados a frutos infestados por $A$. obliqua.

De maneira geral, os frutos do taperebazeiro são desuniformes, apresentando uma considerável variação de tamanho, peso e espessura de polpa. Souza et al. (2000) obtiveram diferenças significativas no peso, comprimento, diâmetro, número de lóculos e de sementes, em amostras de taperebá de cinco plantas, oriundas dos estados do Ceará e da Paraíba. Adicionalmente, Bezerra et al. (2010) verificaram diferenças na composição química de frutos de duas amostras de taperebá oriundas de um único município do estado do Amapá.

Segundo Sivinski et al. (1997), o tamanho dos frutos parece ser a característica determinante para o sucesso do parasitismo. De acordo com estes autores, frutos maiores geralmente apresentam menor percentual de parasitismo, característica aparentemente resultante da proteção recebida pela larva em função da quantidade de polpa e casca presente no fruto. Desta forma, espera-se maior quantidade de parasitóides em frutos menores.

Hickel (2002) coletou amostras de frutos maduros de café (Coffea arabica), jabuticaba (Myrciaria cauliflora), cajámirim (Spondias lutea) e laranja (Citrus aurantium), e concluiu que o nível de parasitismo apresentou uma correlação negativa com a espessura da polpa dos frutos, sendo os frutos de polpa fina mais adequados ao desenvolvimento de parasitóides de moscas-das-frutas, enquanto 
que frutos de polpa espessa tendem a apresentar nível de parasitismo reduzido.

Este trabalho objetivou verificar se a espessura da polpa, o tamanho e o peso de frutos interferem na incidência de parasitóides de moscas-das-frutas.

\section{MATERIAL E MÉTODOS}

Procedeu-se a coleta de 75 frutos maduros de um taperebazeiro com $15 \mathrm{~m}$ de altura, na área urbana do município de Macapá, Amapá $\quad\left(00^{\circ} 00^{\prime} 28,2\right.$ ' 'S; $\left.51^{\circ} 04^{\prime} 48,4^{\prime \prime} \mathrm{W}\right)$, diretamente da planta, no terço inferior da copa ( 4 a $5 \mathrm{~m}$ de altura). Os frutos foram conduzidos ao Laboratório de Entomologia da Embrapa Amapá, onde foram pesados em balança de precisão. $O$ maior e menor diâmetro dos frutos foram tomados com auxílio de paquímetro digital, padronizando o local de tomada da medida, de acordo com o formato do fruto. Os frutos foram individualizados e acondicionados em recipientes plásticos $(8 \mathrm{~cm}$ de diâmetro) cobertos com organza presa com ligas elásticas. Uma fina camada de vermiculita umedecida foi colocada no fundo de cada recipiente, com as funções de manutenção da umidade do fruto e como substrato para pupamento das moscas-das-frutas.

Até 21 dias após a coleta, os substratos foram inspecionados a cada sete dias, sendo os pupários acondicionados em recipientes plásticos contendo vermiculita. $\mathrm{O}$ material foi mantido em câmaras climatizadas sob temperatura $\left(26 \pm 0,5^{\circ} \mathrm{C}\right)$, umidade relativa do ar $(70 \pm 5 \%)$ e fotofase (12 horas), sendo observados diariamente.

Terminado o período para pupamento, a polpa dos frutos foi removida, sendo tomadas as medidas de maior e menor diâmetro dos caroços, com auxílio de paquímetro digital. A espessura da polpa foi obtida pela diferença entre os maiores e menores diâmetros (representados por $\phi$ ) dos frutos e dos caroços, desconsiderando a espessura da casca.

As moscas-das-frutas e os parasitóides emergidos foram fixados em etanol a $70 \%$ e identificados seguindo as chaves de Zucchi (2000) e Canal \& Zucchi (2000).

Foram calculados os índices de infestação dos frutos $(\mathrm{I}=$ número de pupários obtidos/número de frutos coletados e $\mathrm{I}=$ número de pupários obtidos/peso de frutos coletados) e o percentual de parasitismo (IP = número de parasitóides emergidos/número de pupários x 100). Os valores de percentual de parasitismo, sem transformação, foram relacionados com os valores da espessura da polpa dos frutos, mediante aplicação de análises estatísticas descritivas, elaboração de histogramas para avaliar as distribuições das frequiências das amostras, bem como alguns testes de hipóteses de interesse à análise, tais como teste de normalidade de Shapiro-Wilk, teste de independência (Kolmogorov-Smirnov), regressões lineares comparativas e ANOVA (Paternelli \& Mello 2007).

\section{RESULTADOS E DISCUSSÃO}

Os frutos coletados totalizaram 952,7 g com peso médio de $12,7 \pm 0,31 \mathrm{~g}$, variando de 6,6 a $22,7 \mathrm{~g}$. A média para o menor diâmetro dos frutos foi $26,5 \pm 0,24 \mathrm{~mm}(21,4$ a $33,7 \mathrm{~mm}$ ) e para o maior diâmetro foi $36,2 \pm 0,30 \mathrm{~mm}(29,3$ a $43,5 \mathrm{~mm})$. A média para espessura de polpa no semi-eixo menor foi $9,5 \pm 0,14 \mathrm{~mm}(6,7$ a $12,2 \mathrm{~mm})$ e no semieixo maior foi $8,7 \pm 0,14 \mathrm{~mm}(3,6$ a 10,8 $\mathrm{mm})$.

Dos 75 frutos, 57 estavam infestadas por moscas-das-frutas $(76 \%)$, originando 135 pupários, com emergência de 10 moscas e 63 parasitóides $(54,0 \%$ de emergência). A infestação do taperebá por moscas-das-frutas foi de 141,7 pupários/Kg, variando de frutos não infestados a frutos com até 8 pupários. 
Os frutos infestados apresentaram média de 2,37 pupários.

$O$ índice de infestação obtido no presente trabalho (área urbana) foi semelhante ao obtido em áreas periurbanas e rurais dos municípios de Itaubal do Piririm (141,1 pupários/kg) e Macapá (141,8 pupários/kg), por Silva et al. (2007a) e Silva et al. (2005), respectivamente, e inferior ao obtido em áreas periurbanas de Santana (45,7 pupários/kg) e Ferreira Gomes (52,3 pupários $/ \mathrm{kg}$ ), por Silva et al. (2007b) e Silva e Silva (2007), respectivamente.

Foram obtidas duas fêmeas de $A$. obliqua, além de oito machos que, por suas características morfológicas externas, foram considerados pertencentes à mesma espécie. Foram obtidos 63 exemplares de parasitóides de duas espécies de Braconidae: $D$. areolatus (36 exemplares) e O. bellus (27), totalizando índice de parasitismo de $46,6 \%$. Ambas as espécies de parasitóides apresentam maior potencial para atuar na regulação populacional de moscas-das-frutas nas condições do Estado do Amapá. Doryctobracon areolatus é predominante, representando mais de $50 \%$ dos parasitóides coletados em diferentes estudos conduzidos no Amapá (Silva et al. 2007a, 2007b). Embora a diversidade de parasitóides obtida no presente estudo seja conhecida para o Amapá, o parasitismo foi superior ao registrado em outros trabalhos no Estado, cujos índices variaram de $10,5 \%$ a $23,6 \%$, respectivamente nos municípios de Santana (Silva et al. 2007b) e Macapá (Silva et al. 2005). O parasitismo obtido em taperebá foi superior ao obtido por Hickel (2002), que em cajá-mirim (Spondias lutea L.) obteve 2,63 a $10,72 \%$ de parasitismo.

A caracterização dos frutos de taperebá, indicada por histogramas das distribuições de freqüências indica que o peso $(p=0,027)$ e o menor diâmetro $\phi)$ dos frutos $(p=0,013)$ não obedeceram a uma distribuição do tipo normal (Figura 1a e 1c), enquanto que a maior espessura se enquadra numa distribuição normal $(\mathrm{p}=0,98)$ (Figura 1b). Estes dados reforçam a ideia da nãouniformidade dos frutos do taperebazeiro, mesmo oriundos de uma mesma fonte amostral. Na Figura 1 (d, e, f) testes de normalidade foram significativos apenas para o diâmetro maior, indicado pela Figura 1e. As demais não são consideradas como distribuição normal. Neste caso, ocorreu uma situação similar, com uma tendência de decrescimento $\left(R^{2}=0,1078 ; p=0,04267\right)$.

Contudo, a variável independente (log do peso) em relação à variável Eficiência de Parasitismo (= percentual de parasitismo), representada por $\mathrm{R}^{2}$ foi muito menor do que o obtido por Sivinski et al. (1997) $\left(\mathrm{R}^{2}=0,70\right)$.

$\mathrm{Na}$ Figura 2 são mostradas duas regressões lineares entre os diâmetros (Maior e Menor). O gráfico com triângulos azuis, representado pela regressão do diâmetro menor em relação ao peso, melhor explica a variação do peso do que o gráfico de pontos vermelhos representando $o$ diâmetro menor. Isto é, a dimensão melhor correlacionada com o peso é o diâmetro menor $\left(\mathrm{R}^{2}=0,4467\right)$, não o diâmetro maior $\left(\mathrm{R}^{2}=0,3838\right)$. A diferença entre ambos os resultados provavelmente ocorre em virtude da irregularidade geométrica dos frutos (diferenças entre os diâmetros - $\phi$ Maior e фMenor), tanto da polpa quanto da semente.

Como a relação entre estas variáveis não é uma constante, como pode ser verificada na própria distribuição dos tamanhos dos frutos (peso, diâmetro maior, diâmetro menor), há uma tendência de ocorrer o efeito representado pela Figura 2.

Neste sentido, para as condições de trabalho apresentadas nesta pesquisa, recomenda-se que a medida mais interessante para correlacionar com o peso seja o diâmetro menor. E, por analogia, a variável peso poderia ser melhor relacionada com a eficiência de parasitismo, tal como indicado pelas regressões da Figura 2. 


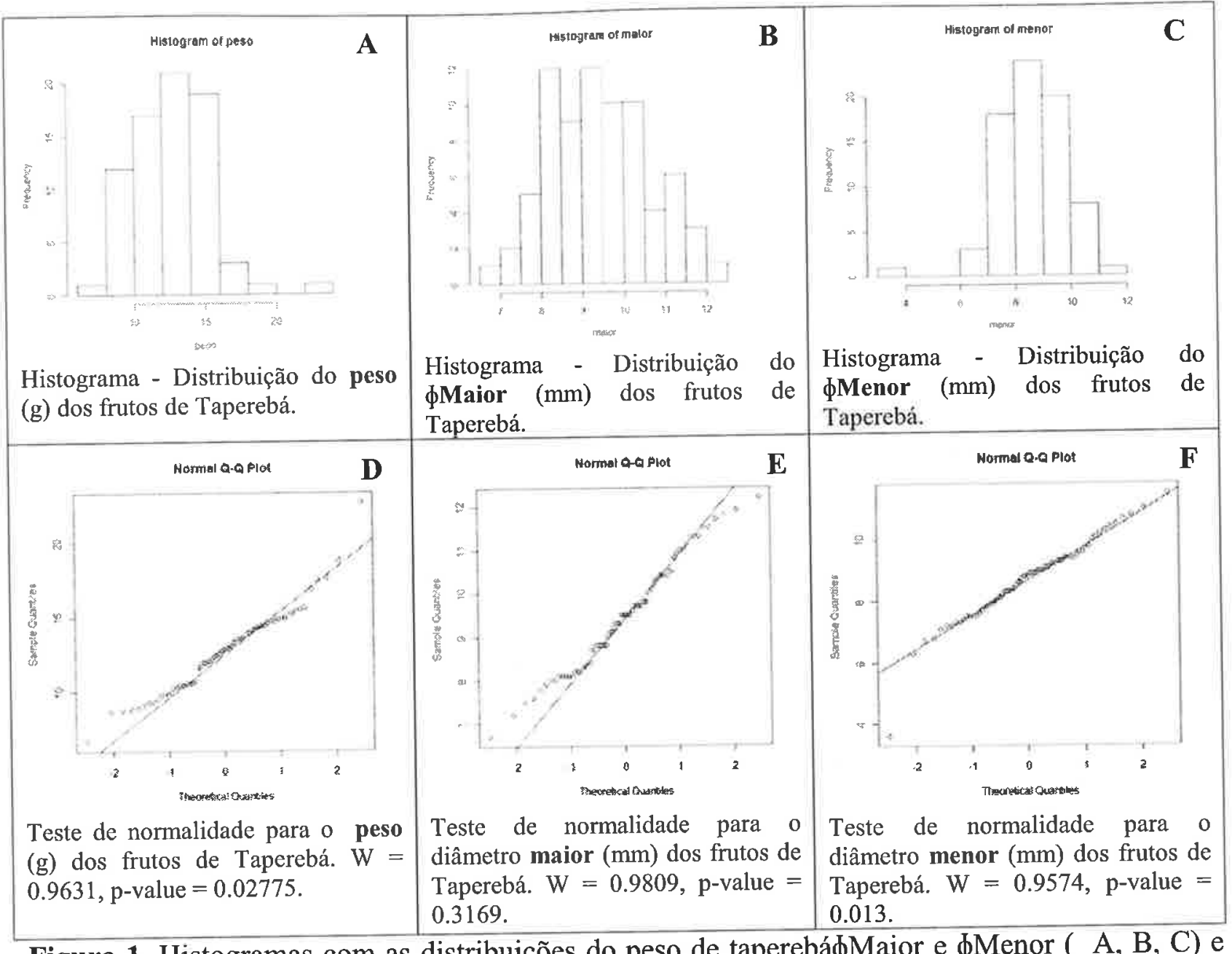

Figura 1. Histogramas com as distribuições do peso de taperebá\$Maior e $\phi$ Menor ( $\mathrm{A}, \mathrm{B}, \mathrm{C}) \mathrm{e}$ testes de normalidade com o método de Shapiro-Wilk (D, E, F), respectivamente. Macapá, abril/2010. 




Figura 2. Correlação entre os diâmetros $\phi$ (Maior - Vermelho e Menor - Azul) e o peso médio dos frutos de taperebá. Macapá, abril/2010.

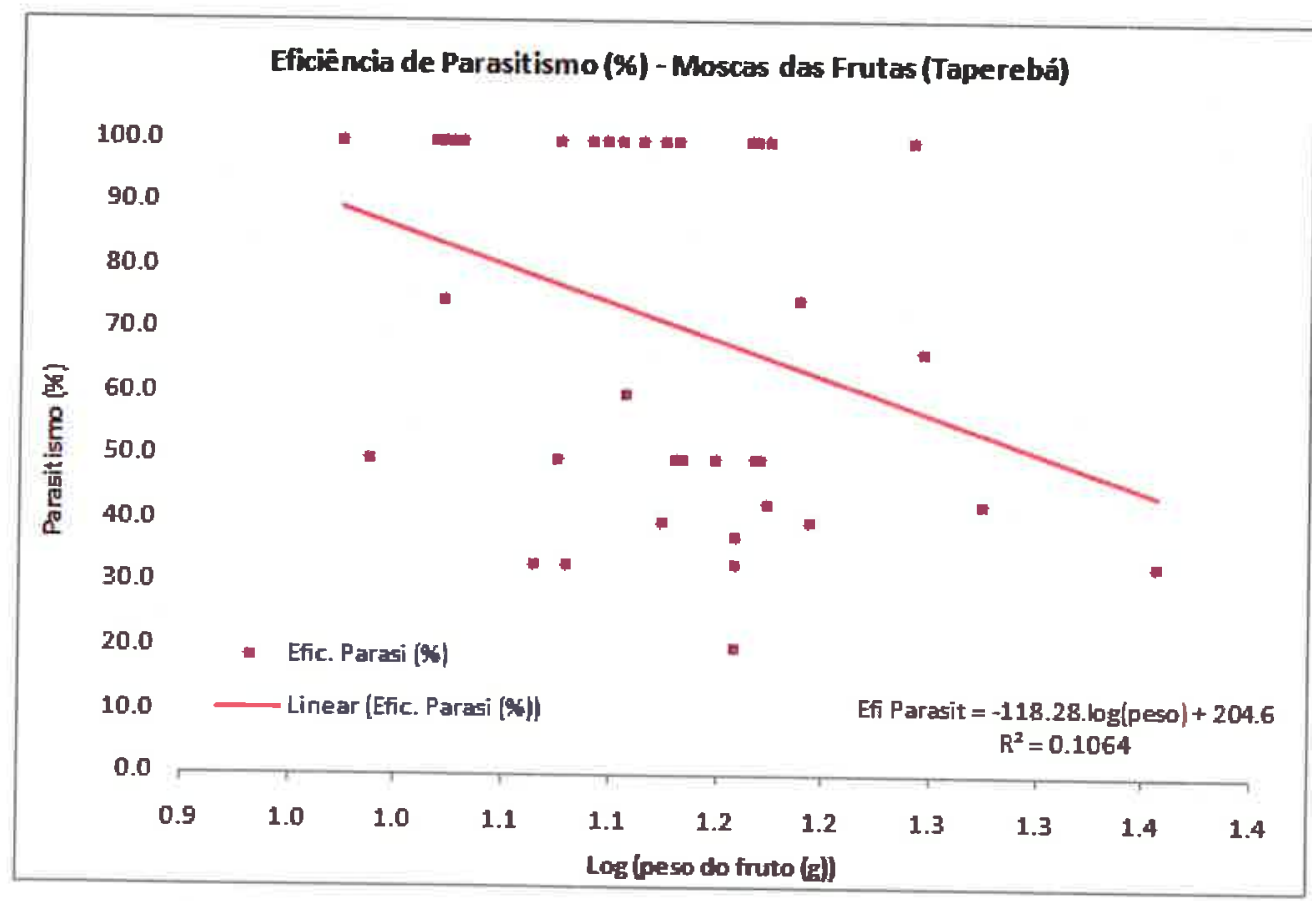

Figura 3. Relação entre a eficiência de parasitismo de Anastrepha em taperebá e o logaritmo do peso médio (só ocorrência positiva). Macapá, abril/2010. 
No entanto, em relação às análises de Sivinski et al. (1997), o presente trabalho apresenta certa similitude comportamental, representado pela tendência de uma curva expressa pela correlação $\log$ do peso $\mathrm{x}$ eficiência do parasitismo (\%) (Figura 3).

Considerando os resultados da presente pesquisa, tal como os indicados pela Figura 2 (relação linear entre os diâmetros e o peso) e pela Figura 3 (relação entre a eficiência de parasitismo e o logaritmo do peso), os resultados não estão compatíveis com os obtidos por Hickel (2002). Tais diferenças parecem estar na metodologia adotada, causadas provavelmente pelo número reduzido de amostras utilizadas por Hickel (2002) e pelas diferenças nos diâmetros das espessuras (maior e menor). Portanto, com os dados obtidos na presente pesquisa não foi possível obter nenhuma correlação funcional semelhante à apresentada por Hickel (2002), do tipo decaimento exponencial, apesar deste referido autor ter utilizado a espessura (não indicou qual delas, se relacionado com o maior ou com o menor diâmetro) ao invés do peso.

As amostras do presente estudo também podem ter induzido o que se denomina de pseudo-repetição amostral, a qual pode não ter permitido a quantificação efetiva da variação da eficiência de parasitismo com o log do peso. Todavia, ao que se observou nesta análise, há uma baixa probabilidade de que as conclusões apresentadas por Hickel (2002) estejam corretas ou sejam generalizadas, pois seus resultados não apresentam a equação de regressão, $\mathrm{R}^{2}$, ou quaisquer outros parâmetros estatísticos comparativos.

Por outro lado, não houve correlação significativa em relação ao percentual de parasitismo versus peso de frutos de taperebá (Figura 3). Parece que as espessuras dos frutos do taperebá não influenciam significativamente o parasitismo ou a eficiência dos parasitóides em encontrarem seus hospedeiros (larvas de moscas). Neste caso, o $\mathrm{R}^{2}$ da curva gerada entre parasitismo e a espessura do diâmetro maior foi da ordem de $\mathrm{R}^{2}=0,0135(\mathrm{Efi}=0,003$ \$Maior $+9,3477)$. Ou seja, praticamente nenhuma correlação entre ambas as variáveis. Em relação ao diâmetro menor, o $\mathrm{R}^{2}$ da curva gerada entre parasitismo e a espessura do diâmetro menor foi da ordem de $\mathrm{R}^{2}=0,083$ $($ Efi $=0,008 \phi$ Menor $+8,3461)$. Ou seja, apesar de um pouco mais significativo em representar o parasitismo, não demonstra quase nenhuma correlação entre ambas as variáveis.

Sivinski et al. (1997) afirmam que nem sempre a relação de interdependência entre o peso do fruto (ou espessura da polpa) e o parasitismo ocorre para todas as situações. Isso significa que é provável que haja outras variáveis independentes (não estudadas nesta pesquisa) que, conjuntamente, expliquem com mais clareza essa relação. Dessa forma, há necessidade de mais estudos para avaliar como a eficiência de parasitismo varia em função de variáveis independentes facilmente mensuráveis, tais como o peso do fruto e o seu respectivo diâmetro mais representativo (diâmetro menor). Nas condições da Amazônia Legal, o ideal é que tais estudos sejam realizados levando-se em consideração as peculiaridades de solo, clima e vegetação que caracterizam os diferentes Estados que compõem a região.

\section{AGRADECIMENTOS}

Ao Conselho Nacional de Desenvolvimento Científico e Tecnológico, pelas Bolsas de Produtividade em Pesquisa concedidas a A. C. Cunha e R. A. Silva. À Coordenação de Aperfeiçoamento de Pessoal de Nível Superior, pela bolsa de pósdoutorado concedida a R. S. Santos. 


\section{REFERÊNCIAS BIBLIOGRÁFICAS}

BEZERRA, V.S., BARROS NETO, E.L., SILVA, R.A. 2010. Características fisicoquímicas de frutos de taperebá (Spondias mombin L.) coletados em área de ocorrência de mosca-das-frutas. In: JORNADA NACIONAL DA AGROINDÚSTRIA, 4., Bananeiras. Anais da IV Jornada Nacional da Agroindústria. Bananeiras- $\mathrm{PB}$ : UFPB/CCHSA/PRAC.

CANAL, N.A., ZUCCHI, R.A. 2000. Parasitóides - Braconidae. In: Malavasi, A. \& Zucchi, R.A. (eds.). Moscas-dasfrutas de importância econômica no Brasil: conhecimento básico e aplicado. Ribeirão Preto: Holos, p.119-126.

HICKEL, E.R. 2002. Espessura da polpa como condicionante do parasitismo de mosca-das-frutas (Diptera: Tephritidae) por Hymenoptera: Braconidae. Ciência Rural, v.32, n.6, p.1005-1009.

LEMOS, L.N., SILVA, R.A., JESUS, C.R., SILVA, W.R., DEUS, E.G., NASCIMENTO, D.B., SOUZA FILHO, M.F. 2008. Índice de infestação de taperebá (Spondias mombin) por Anastrepha spp. (Dip., Tephritidae) em quatro municípios do Estado do Amapá. In: CONGRESSO BRASILEIRO DE ENTOMOLOGIA, 22., Uberlândia. Ciência, Tecnologia e Inovação. Anais... Viçosa, MG: UFV, 1 CD-ROM.

LORENZI, H., BACHER, L., LACERDA, M., SARTORI, S. 2006. Frutas brasileiras e exóticas cultivadas: de consumo in natura. São Paulo: Instituto Plantarum de Estudos da Flora. 640p.

PATERNELLI, L.A., MELLO, M.P. 2007.

Conhecendo o R: uma visão estatística. Viçosa: Ed UFV. 181p.

SILVA, R.A., SILVA, W.R., NASCIMENTO, D.B., SILVA, C.A. 2005. Levantamento de moscas-das frutas e seus parasitóides em frutos de taperebazeiro na Área de Proteção
Ambiental do Rio Curiaú, Macapá, AP. Macapá: Embrapa Amapá, 6p. (Embrapa Amapá. Comunicado Técnico, 116).

SILVA, R.A., DEUS, E.G., PEREIRA, J.D.B., JESUS, C.R., SOUZA-FILHO, M.F., ZUCCHI, R.A. 2011. Conhecimento sobre moscas-das-frutas no Estado do Amapá. In: Silva, R.A., Lemos, W.P., Zucchi, R.A. (Ed. Técn.) Moscas-das-frutas na Amazônia brasileira: diversidade, hospedeiros e inimigos naturais. Macapá: Embrapa Amapá.

SILVA, R.A., NASCIMENTO, D.B., DEUS, E.G., SOUZA, G.D., OLIVEIRA, L.S.P. 2007a. Hospedeiros e parasitóides de Anastrepha spp. (Diptera: Tephritidae) em Itaubal do Piririm, Estado do Amapá. Ciência Rural, v.37, p.557-560.

SILVA, R.A., XAVIER, S.L.O,, SOUZAFILHO, M.F., SILVA, W.R., NASCIMENTO, D.B., DEUS, E.G. 2007b. Frutíferas hospedeiras e parasitóides (Hym., Braconidae) de Anastrepha spp. (Dip., Tephritidae) na Ilha de Santana, Estado do Amapá, Brasil. Arquivos do Instituto Biológico, São Paulo, v.74, p.153-156,

SILVA, W.R., SILVA, R.A. 2007. Levantamento de moscas-das-frutas e de seus parasitóides no Município de Ferreira Gomes, Estado do Amapá. Ciência Rural, Santa Maria, v.37, p.265268.

SIVINSKI, J., ALUJA, M., LOPEZ, M. 1997. Spatial and temporal distributions of parasitoids of mexican Anastrepha species (Diptera:Tephritidae) within the canopies of fruit trees. Annals of the Entomological Society of America, v.90, p.604-618.

SOUZA, F.X., SOUSA, F.H.L., FREITAS, J.B.S., ROSSETTI, A.G. 2000. Aspectos morfológicos da unidade de dispersão de cajazeira. Pesquisa Agropecuária Brasileira, v.35, p.215-220. 
Efeito da espessura da polpa, tamanho e peso de frutos de taperebá (Spondias mombin L.) sobre o parasitismo natural (Hymenoptera: Braconidae) em moscas-das-frutas (Diptera: Tephritidae)

ZUCCHI, R.A. 2000. Taxonomia. In: Malavasi, A. \& Zucchi, R.A. (eds.). Moscas-das-frutas de importância econômica no Brasil: conhecimento básico e aplicado. Ribeirão Preto: Holos, p.13-24.
ZUCCHI, R.A. 2008. Fruit flies in Brazil Anastrepha species their host plants and parasitoids. Disponível

em: www.lea.esalq.usp.br/anastrepha/, atualizado em 19 de janeiro de 2011. Acesso em: 15/04/2011. 